\title{
New Tunneling Features in Polar III-Nitride Resonant Tunneling Diodes
}

\begin{abstract}
Jimy Encomendero, ${ }^{1, *}$ Faiza Afroz Faria, ${ }^{2}$ S. M. Islam, ${ }^{1}$ Vladimir Protasenko, ${ }^{1}$ Sergei Rouvimov, ${ }^{2}$ Berardi Sensale-Rodriguez, ${ }^{3}$ Patrick Fay, ${ }^{2}$ Debdeep Jena, ${ }^{1,4, \uparrow}$ and Huili Grace Xing ${ }^{1,4,5, \sharp}$

${ }^{1}$ School of Electrical and Computer Engineering, Cornell University, Ithaca, New York 14853, USA

${ }^{2}$ Department of Electrical Engineering, University of Notre Dame, Notre Dame, Indiana 46556, USA

${ }^{3}$ Department of Electrical and Computer Engineering, The University of Utah, Salt Lake City, Utah 84112, USA

${ }^{4}$ Department of Materials Science and Engineering, Cornell University, Ithaca, New York 14853, USA

${ }^{5}$ Kavli Institute at Cornell for Nanoscale Science, Cornell University, Ithaca, New York 14853, USA

(Received 7 February 2017; revised manuscript received 4 July 2017; published 23 October 2017)

For the past two decades, repeatable resonant tunneling transport of electrons in III-nitride double barrier heterostructures has remained elusive at room temperature. In this work we theoretically and experimentally study III-nitride double-barrier resonant tunneling diodes (RTDs), the quantum transport characteristics of which exhibit new features that are unexplainable using existing semiconductor theory. The repeatable and robust resonant transport in our devices enables us to track the origin of these features to the broken inversion symmetry in the uniaxial crystal structure, which generates built-in spontaneous and piezoelectric polarization fields. Resonant tunneling transport enabled by the ground state as well as by the first excited state is demonstrated for the first time over a wide temperature window in planar III-nitride RTDs. An analytical transport model for polar resonant tunneling heterostructures is introduced for the first time, showing a good quantitative agreement with experimental data. From this model we realize that tunneling transport is an extremely sensitive measure of the built-in polarization fields. Since such electric fields play a crucial role in the design of electronic and photonic devices, but are difficult to measure, our work provides a completely new method to accurately determine their magnitude for the entire class of polar heterostructures.
\end{abstract}

DOI: 10.1103/PhysRevX.7.041017

Subject Areas: Condensed Matter Physics,

Electronics, Semiconductor Physics

\section{INTRODUCTION}

Resonant tunneling of electrons in III-V semiconductors has been extensively studied since Tsu and Esaki theoretically investigated tunneling transport across multibarrier heterostructures with periods comparable to the electron wavelength [1]. Quantum confinement introduced by the barriers results in a localized and discrete electronic energy spectrum, which can be tuned to bring the bound states in resonance with any adjacent reservoir of electrons.

The resonant transport regime has been exploited to design highly efficient injectors of electrons into the upper lasing level of terahertz (THz) quantum cascade lasers (QCLs) [2]. However, THz QCLs fabricated with the well-developed material systems $\mathrm{AlGaAs} / \mathrm{GaAs}$ and $\mathrm{InGaAs} / \mathrm{InAlAs}$ have not yet achieved room-temperature (RT) operation and their

\footnotetext{
*jje64@ cornell.edu

†jena@cornell.edu

grace.xing@cornell.edu
}

Published by the American Physical Society under the terms of the Creative Commons Attribution 4.0 International license. Further distribution of this work must maintain attribution to the author(s) and the published article's title, journal citation, and DOI. lasing frequencies are limited to less than $5 \mathrm{THz}$ mainly due to Reststrahlen absorption [3].

On the other hand, the out-of-resonance regime, identified by the onset of negative differential conductance (NDC), has been harnessed to realize high-frequency resonant tunneling diode (RTD) oscillators [4]. Despite the steady progress in output power and frequency of operation, RTD oscillators are yet to be demonstrated at the milliwatt output power level for frequencies higher than $1 \mathrm{THz}$, which is required for most practical applications [5].

In this scenario, the GaN/AlGaN material system has emerged as an attractive alternative to realize intersubband emitters within a wide range of frequencies due to the large conduction band energy offset $\Delta E_{c} \approx 1.75 \mathrm{eV}$ between GaN and AlN $[6,7]$. In addition, the high longitudinal optical (LO) phonon energy of III-nitride materials- $\hbar \omega_{\mathrm{LO}} \approx 92 \mathrm{meV}$ in $\mathrm{GaN}$ - is expected to prevent the depopulation of the upper lasing level, thus raising hopes for RT operation of nitride THz QCLs [8].

Double-barrier $\mathrm{GaN} / \mathrm{Al}(\mathrm{Ga}) \mathrm{N}$ RTDs, being the simplest device to study resonant transport, have been under scrutiny during the past decades with moderate success [9-23]. Experiments on AlN-barrier RTDs grown on sapphire templates have shown a region of NDC under forward bias (the bottom contact layer is the reference of the applied 
voltage). However, these devices present nonrepeatable current-voltage $(I-V)$ characteristics [9-12]. It has been suggested that the high density of defects present in $\mathrm{GaN}$ films grown on sapphire (with a typical dislocation density of $10^{9} \mathrm{~cm}^{-2}$ ) can act as electron traps, leading to selfcharging effects and preventing coherent transport of carriers.

It should be pointed out, however, that repeatable roomtemperature NDC was reported in a multiple quantum well heterostructure lattice matched to an AlN film grown on sapphire [24]. In this device, multiple barriers were employed to significantly reduce the leakage current across the active region. As a consequence, multiple resonances were observed at room temperature due to the energy alignment of the various quantum states localized within adjacent wells. However, the strong attenuation introduced by the multiple tunneling barriers resulted in a modest peak current density of $\sim 4.2 \mathrm{~mA} / \mathrm{cm}^{2}$.

Other approaches have also been employed to significantly reduce the defect densities and enhance resonant transport in double-barrier resonant tunneling heterostructures. Among them, low-temperature grown superlattices [13] and lateral epitaxial overgrowth [14] were employed to reduce the number of dislocations across the active region; however, the NDC still degraded with consecutive measurements [15]. III-nitride nanowires were also investigated as a means to obtain defect-free double-barrier heterostructures. Multiple resonances were observed in these one-dimensional heterostructures at cryogenic temperatures [16]. Later, Shao et al. reported room-temperature resonances, which disappeared in subsequent measurements [17].

More recently, III-nitride RTDs with low Al-composition AlGaN barriers have also been studied at cryogenic temperatures [25-27]. These devices, grown on freestanding GaN substrates (with low dislocation densities $\sim 5 \times 10^{6} \mathrm{~cm}^{-2}$ ), feature small mesa areas of $\sim 16 \mu \mathrm{m}^{2}$ aimed towards minimizing the number of defects per device. Peak current densities up to $\sim 375 \mathrm{kA} / \mathrm{cm}^{2}$ and repeatable NDC were observed only at cryogenic temperatures: below $110 \mathrm{~K}$ for a diode with $18 \% \mathrm{AlGaN}$ barriers and below $130 \mathrm{~K}$ for a diode with $35 \% \mathrm{AlGaN}$ barriers. It has been only recently that repeatable room-temperature NDC was reported in GaN/AIN RTDs by us [28] and by Growden et al. [29], an achievement under the ONR DATE MURI program.

In this work, GaN/AlN RTDs are studied both experimentally and theoretically to understand the fundamental role played by the spontaneous and piezoelectric polarization fields in the device electrostatics and quantum transport. In the next section, we provide details of the growth, surface morphology, and structure of a doublebarrier RTD sample. We then present a comprehensive characterization study in terms of repeatable operation, temperature-dependent tunneling transport, and structural analysis of the fabricated device. After introducing an analytical model for polar RTDs, its main predictions are experimentally verified by fabricating a series of RTDs in which the tunneling barrier thickness is systematically varied. Room-temperature $I-V$ measurements show a clear resonant peak and repeatable NDC under forward bias in each of the fabricated samples. This repeatable operation measured across different device designs has helped uncover several unique features present in polar RTDs and demonstrate that III-nitride heterostructures are capable of robust resonant tunneling transport at room temperature.

\section{EXPERIMENT}

Symmetric double-barrier GaN/AIN heterostructures are grown by molecular beam epitaxy (MBE) on the $c$ plane of commercially available $n$-type bulk GaN substrates with a nominal dislocation density $\sim 5 \times 10^{4} \mathrm{~cm}^{-2}$. Metal-rich growth conditions are maintained during the whole process at a substrate temperature of $\sim 700^{\circ} \mathrm{C}$ and a nitrogen plasma power of $\sim 200 \mathrm{~W}$. A growth rate of $\sim 3 \mathrm{~nm} / \mathrm{min}$ is estimated by post-growth structural characterization methods. The active region of the heterostructure, shown in Fig. 1(a), comprises a $t_{w} \approx 3$-nm-wide GaN quantum well and AlN barriers of thickness $t_{b} \approx 2 \mathrm{~nm}$. Unintentionally doped (UID) spacers of thickness $t_{s} \approx 2 \mathrm{~nm}$ are also grown next to both barriers. The emitter and collector regions consist of $\sim 100$-nm $n$-type GaN layers with a silicon donor doping level $N_{d} \approx 7 \times 10^{18} \mathrm{~cm}^{-3}$. A highly Si-doped $\left(N_{d}^{+} \approx 8 \times 10^{19} \mathrm{~cm}^{-3}\right) \mathrm{GaN}$ cap layer completes the epitaxial heterostructure allowing for the formation of Ohmic contact electrodes.

MBE growth of GaN films has been studied extensively in recent years, showing that smooth surfaces can be generated under metal-rich growth conditions. In this regime, a Ga adlayer of $\sim 2.5$ monolayers (ML) is present at the interface favoring adatom mobility and improving the surface morphology [30-32]. Similar conditions are employed throughout the growth of the emitter and collector $n$-GaN layers as well as during the incorporation of the GaN quantum well. However, using excess $\mathrm{Al}$ metal during the growth of the AlN barriers leads to thicker layers since the excess $\mathrm{Al}$ readily incorporates into the crystal in preference to $\mathrm{Ga}$ [33]. An alternative approach exploits the surfactant effects of excess $\mathrm{Ga}$, which decreases the surface energy without getting incorporated into the AlN films [32]. Therefore, in this work, Ga-rich growth conditions within the step-flow growth mode are employed for the epitaxy of the different device structures. Maintaining these conditions has proven to be critical for achieving atomically smooth interfaces and minimizing the formation of defects, which is crucial for resonant tunneling transport.

Atomic force microscopy is employed to image the surface of the as-grown sample, revealing a surface morphology composed by 2-ML atomic steps with a root-mean-square (rms) roughness of $\sim 0.146 \mathrm{~nm}$ over an 
(a)

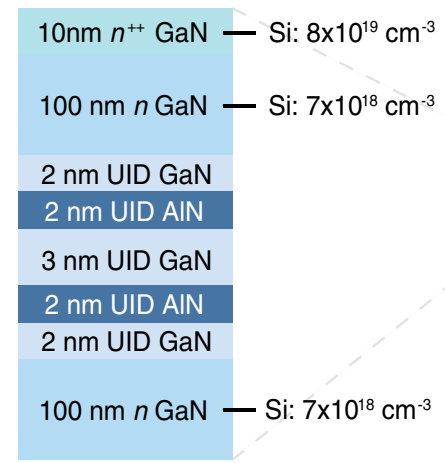

(b)

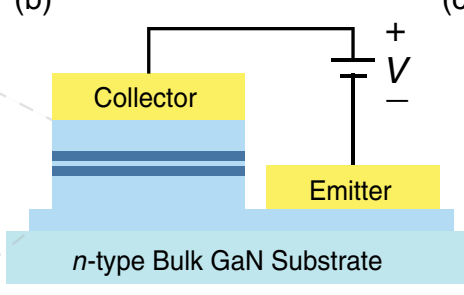

(c)

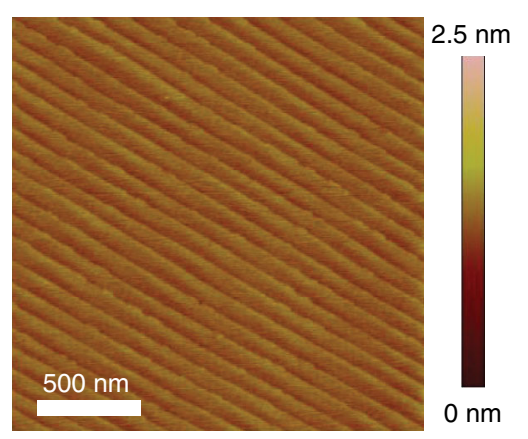

FIG. 1. (a) Schematic of the device structure and doping concentrations of the GaN/AIN double-barrier heterostructure grown by MBE on Ga-polar single-crystal GaN substrates. (b) Cross-section schematic diagram of a fabricated resonant tunneling diode with the collector and emitter metal contacts under forward bias. (c) Surface morphology of the as-grown RTD heterostructure with a rms roughness of $\sim 0.146 \mathrm{~nm}$ over a $2 \times 2 \mu \mathrm{m}^{2}$ area.

area of $2 \times 2 \mu \mathrm{m}^{2}$ [Fig. 1(c)]. Finally, it should be noted that crystal defects stemming from strain relaxation processes should be suppressed since the AIN barriers are thinner than the experimentally identified critical thickness $(\sim 5-7 \mathrm{~nm})$ for films pseudomorphically grown on GaN [34] and [35].

The RTD fabrication is carried out using conventional contact photolithography, electron beam evaporation, and reactive ion etching techniques. The collector contact is defined by evaporating a $\mathrm{Ti} / \mathrm{Al} / \mathrm{Au} / \mathrm{Ni}$ metal stack. Mesas with areas between 6 and $48 \mu \mathrm{m}^{2}$ are defined using a selfaligned process in which the structure is etched down to the emitter $n$-GaN layer by reactive ion etching in a $\mathrm{Ar} / \mathrm{Cl}_{2} / \mathrm{BCl}_{3}$ gas mixture. Finally, the emitter $\mathrm{Ti} / \mathrm{Al} / \mathrm{Au}$ metal stack is deposited to obtain the structure depicted in Fig. 1(b). Transfer length method structures are also defined; a specific contact resistance of $\sim 30 \Omega \mu \mathrm{m}^{2}$ is measured from the collector contacts, and the emitter contact resistance is estimated to be of the same order of magnitude. Thus, under injection currents on the order of $\sim 10 \mathrm{kA} / \mathrm{cm}^{2}$, a voltage drop of $\sim 3 \mathrm{mV}$ per contact is expected, which is negligible compared to the total voltage used to bias the diodes.

\section{RESULTS}

Current-voltage characteristics are measured with a semiconductor parameter analyzer at room temperature applying voltage sweeps starting at 0 up to $16 \mathrm{~V}$, and then back to $0 \mathrm{~V}$ (double sweep). Immediately after the forward bias measurement, a reverse bias double sweep with a minimum voltage of $-4.4 \mathrm{~V}$ is also performed, completing a closed-loop scan [bottom inset of Fig. 2(a)]. The polarity of the diode bias is that of the voltage applied to the collector side, having the emitter as reference, as shown in Fig. 1(b).

During the first forward scan and for voltages below $5 \mathrm{~V}$, current densities below $10 \mathrm{~A} / \mathrm{cm}^{2}$ are measured [Fig. 2(a)]. These low injection currents are a result of the large
GaN/AlN conduction band discontinuity $(\sim 1.75 \mathrm{eV})$ and the presence of polarization fields that effectively block carrier transport. The energy band diagram shown in Fig. 2(b), calculated using a self-consistent SchrödingerPoisson solver [36], illustrates this blocking effect at equilibrium conditions.

A resonant peak is measured in the first upward sweep at $\sim 10.7 \mathrm{~V}$ with a peak current density of $\sim 5.7 \mathrm{kA} / \mathrm{cm}^{2}$, as shown in Fig. 2(a). The adjacent NDC region extends up to $\sim 11.5 \mathrm{~V}$ where the valley current is measured at the level of $\sim 3.7 \mathrm{kA} / \mathrm{cm}^{2}$. For biases larger than $12 \mathrm{~V}$, the current increases exhibiting an exponential trend consistent with thermionic emission over the barriers. The resonant peak is attributed to the enhancement of transmission probability for carriers populating the accumulation subband adjacent to the emitter AlN barrier. The band diagram corresponding to this resonant condition is plotted in Fig. 2(c).

During the downward sweep of the first closed-loop scan, a shift in the resonant voltage is measured [Fig. 2(a)]. This shift points to the presence of trap charging mechanisms, which modify the electrostatic profile of the heterostructure. A detailed study of the dynamics, activation energy, and location of the traps is beyond the scope of this work. Nevertheless, despite the presence of these crystal imperfections in this particular sample, resonant transport of electrons remains the main conduction mechanism at room temperature evidenced by the presence of the resonant peak and NDC in both upward and downward sweeps. Furthermore, it should be noted that the resonant peak and NDC are observed at room temperature over the full range of fabricated mesa areas which span from 6 up to $48 \mu \mathrm{m}^{2}$. Meanwhile, the magnitude of RTD tunneling current scales with the different mesa areas. This observation suggests that sidewall leakage current does not play a significant role in the device characteristics.

When the diodes are biased in the reverse polarity, a region of low injection currents $\left(<10 \mathrm{~A} / \mathrm{cm}^{2}\right)$ is also measured [Fig. 2(a)]. However, a transition to a higher current injection regime can be seen around the reverse 

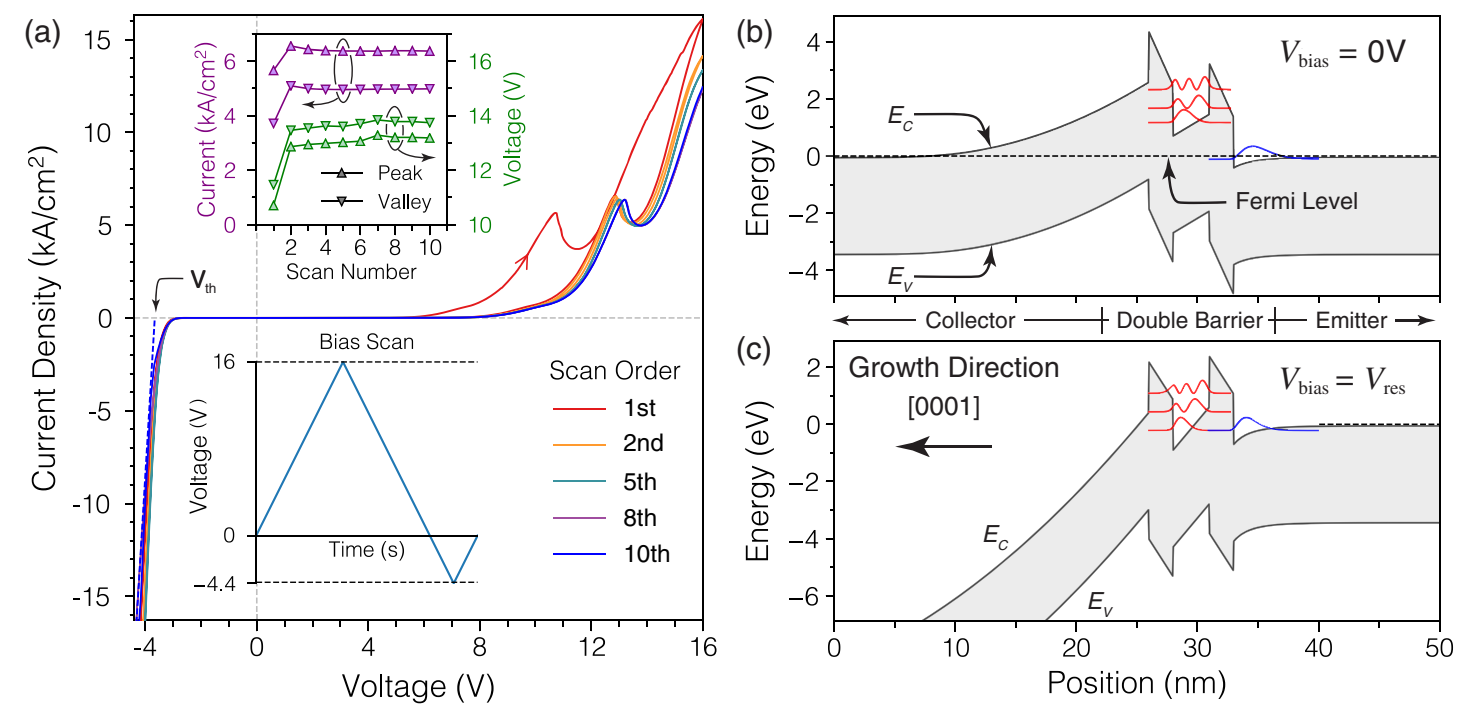

FIG. 2. (a) Current-voltage characteristics of GaN/AlN RTDs with their layer structure shown in Fig. 1(a). The measurements, performed at room temperature, were obtained by applying closed-loop bias scans as shown in the bottom inset. Subsequent closed-loop measurements were performed on the same device featuring a mesa area of $\sim 24 \mu \mathrm{m}^{2}$. A shift in the peak voltage is observed during the first closed-loop scan; however, it disappears in the following scans. The onset of NDC stabilizes at $\sim 13.2 \mathrm{~V}$ with a repeatable peak current density of $\sim 6.4 \mathrm{kA} / \mathrm{cm}^{2}$ [top inset of (a)]. The stable RTD characteristics confirm the presence of resonant tunneling transport at room temperature with a minimum NDC of $\sim-5.9 \mathrm{kS} / \mathrm{cm}^{2}$. (b) Band diagram under equilibrium conditions showing the accumulation subband in the emitter region and resonant states in the quantum well. (c) Band diagram under resonant conditions enabled by the energy alignment between the accumulation subband next to the emitter AlN barrier and the ground state of the quantum well.

threshold voltage $V_{\text {th }}=-3.6 \mathrm{~V}$, after which the current increases monotonically, reaching a level of $\sim 15 \mathrm{kA} / \mathrm{cm}^{2}$ at $-4.4 \mathrm{~V}$. This behavior contrasts with the $\sim 16 \mathrm{~V}$ required to achieve similar current levels in the forward direction. This asymmetric $I-V$ is a direct consequence of the polarization electric fields present in the heterostructure, due to broken inversion symmetry of the uniaxial crystal. This interesting connection is discussed in detail in the next section.

Subsequent closed-loop bias scans are performed in the same device as shown in Fig. 2(a). It is found that the resonant bias increases slightly with additional scans and eventually stabilizes at $\sim 13.2 \mathrm{~V}$ for both upward and downward sweeps [top inset of Fig. 2(a)]. The peak-tovalley current ratio (PVCR) decreases from its initial value of $\sim 1.5$ during the first scan to a stable value of $\sim 1.3$ in the eighth and subsequent scans. This trend is caused by the larger increase of the valley current which experiences a $\sim 25.5 \%$ increment with respect to its initial value in the first scan, whereas the peak current increases only $\sim 11.1 \%$. This behavior suggests the presence of defects in the AlN barriers in this sample that act as current leakage paths and degrade the energy filtering mechanism of the doublebarrier heterostructure. It should be noted, however, that these leakage paths exhibit limited effects and do not prevent resonant tunneling transport of carriers across the active region at room temperature.

Repeatable room-temperature NDC has also been recently demonstrated in a similar heterostructure grown at a higher growth temperature [29]. A very small voltage shift attributed to Joule heating was measured in those devices. These results suggest that traps giving rise to the voltage shift in the first scan [Fig. 2(a)] could be minimized by enhancing adatom mobilities during the epitaxial growth. In contrast, our devices exhibit a larger PVCR, potentially due to the sharp interfaces in the active region (see Fig. 4 and the last paragraph in this section). In this sense, maintaining an optimal growth temperature and forming atomically smooth interfaces is key towards the realization of room-temperature RTDs. We systematically investigated the impact of the growth temperature on resonant tunneling transport in nitride RTDs; this experimental study will be published separately. Nonetheless, it is worth noting that all the subsequent GaN/AlN RTDs grown under the improved growth conditions exhibit stable NDC at room temperature from the first scan, i.e., no shift in the $I-V$ curves. These findings suggest that defects in the RTD structures are sufficiently suppressed.

Temperature-dependent $I-V$ measurements are also performed in our devices down to $4.2 \mathrm{~K}$ using liquid helium. Figures 3(a) and 3(b) show the current-voltage characteristics for a particular device featuring an area of $12 \mu \mathrm{m}^{2}$. Various conduction regimes can be clearly identified as the current components exhibit different behavior as a function of temperature. Transport under low forward bias $(<5 \mathrm{~V})$ is supported mainly by thermionic emission over the doublebarrier active region. In this regime, the RTD current exhibits a strong temperature dependence clearly visible 

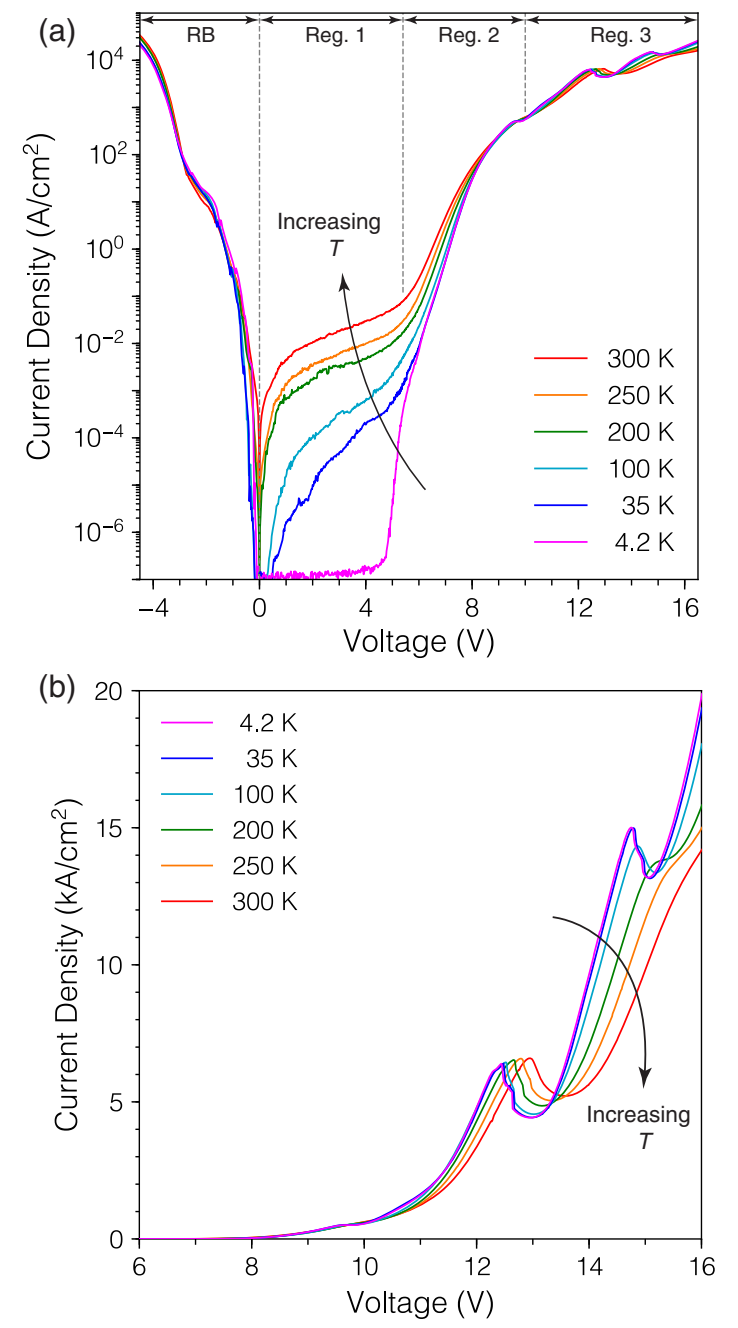

FIG. 3. (a) Semilogarithmic $I-V$ plot showing regions with different transport regimes as a function of temperature: Under reverse bias (RB), the current exhibits a weak temperature dependence. In contrast, within region 1 a strong temperature dependence is measured since the current is enabled mainly by thermionic electrons. Region 2 presents a weak dependence on temperature due to the enhancement of the tunneling current component. Transport within region 3 is mainly due to resonant tunneling of carriers. (b) Forward $I$ - $V$ curves at different temperatures showing the repeatable room-temperature resonant peak and a higher-order resonance for temperatures below $200 \mathrm{~K}$.

in region 1 of Fig. 3(a). As the bias is increased ( $>5 \mathrm{~V})$, the resonant levels of the GaN quantum well shift towards lower energies and get closer to the Fermi level of the emitter region. The energy alignment between the ground state of the well and the quasicontinuous scattering states within the emitter region give rise to an increment in the current, which is supported by carriers tunneling across the AlN barriers. This conduction enhancement due to the onset of tunneling constitutes one of the main transport mechanisms within region 2 of Fig. 3(a) and it is confirmed by the weak temperature dependence of the current. At $\sim 10 \mathrm{~V}$, a transition is observed, evidenced by the conductance behavior that initially decreases slightly and then increases again. This modulation is explained as the onset of out-of-resonance conditions between the quasicontinuous scattering states of the emitter and the ground state of the well. In this regime, the tunneling nature of the diode current is confirmed by the clear temperature independence of its magnitude at $\sim 10 \mathrm{~V}$ [Fig. 3(b)]. Studies done in GaAs/AlGaAs RTDs featuring low-doped emitters have also reported a similar conduction regime manifested by a slight modulation of the current in the rising side of the main resonant peak [37,38]; this is the first time that this behavior is encountered in nitride RTDs.

When a voltage larger than $10 \mathrm{~V}$ is applied, various resonances are measured both at room temperature as well as at cryogenic temperatures [Fig. 3(b)]. The first resonant peak is attributed to the tunneling coupling between the accumulation subband [bound state situated below the quasicontinuous scattering states of the emitter; see Fig. 2(c)] and the ground state of the quantum well. As the temperature is reduced, the resonant voltage shifts towards lower values for both resonances, most probably due to a reduction in the series resistance of the device. Furthermore, the peak current density of the first resonance is nearly independent of temperature, as expected from its resonant tunneling origin. The PVCR increases up to $\sim 1.4$ at $4.2 \mathrm{~K}$ from its room-temperature value of $\sim 1.3$. This results from the reduction of the valley current whose magnitude decreases as scattering processes are suppressed at lower temperatures. At room temperature, the first NDC region exhibits a minimum differential conductance of $\sim-5.9 \mathrm{kS} / \mathrm{cm}^{2}$. As the temperature is lowered, the negative conductance increases up to a point where selfoscillations start to build up.

A higher-order resonance is also measured at cryogenic temperatures, as shown in Fig. 3(b). This resonant feature is measured clearly at temperatures below $200 \mathrm{~K}$, exhibiting a maximum peak current density of $\sim 15 \mathrm{kA} / \mathrm{cm}^{2}$ at $4.2 \mathrm{~K}$. It is suggested that this additional peak arises from resonant tunneling transport of carriers via the first excited state of the quantum well. The magnitude of the valley current decreases as the temperature is lowered and a maximum PVCR of $\sim 1.1$ is measured at the lowest temperature. On the other hand, the peak current density increases with decreasing temperature. This behavior has been reported previously in GaAs-based resonant tunneling devices [39]. Its origin stems mainly from the thermal population of the electronic states around the Fermi level. At lower temperatures, a higher resonant current is expected since the emitter states aligned with the bound state exhibit higher populations [40]. Shen et al. developed a model that takes into account these effects and confirmed the origin of the resonant current enhancement in GaAs RTDs [41]. In addition, a similar behavior has been recently observed in the peak current of 2D crystal Esaki diodes [42]. 

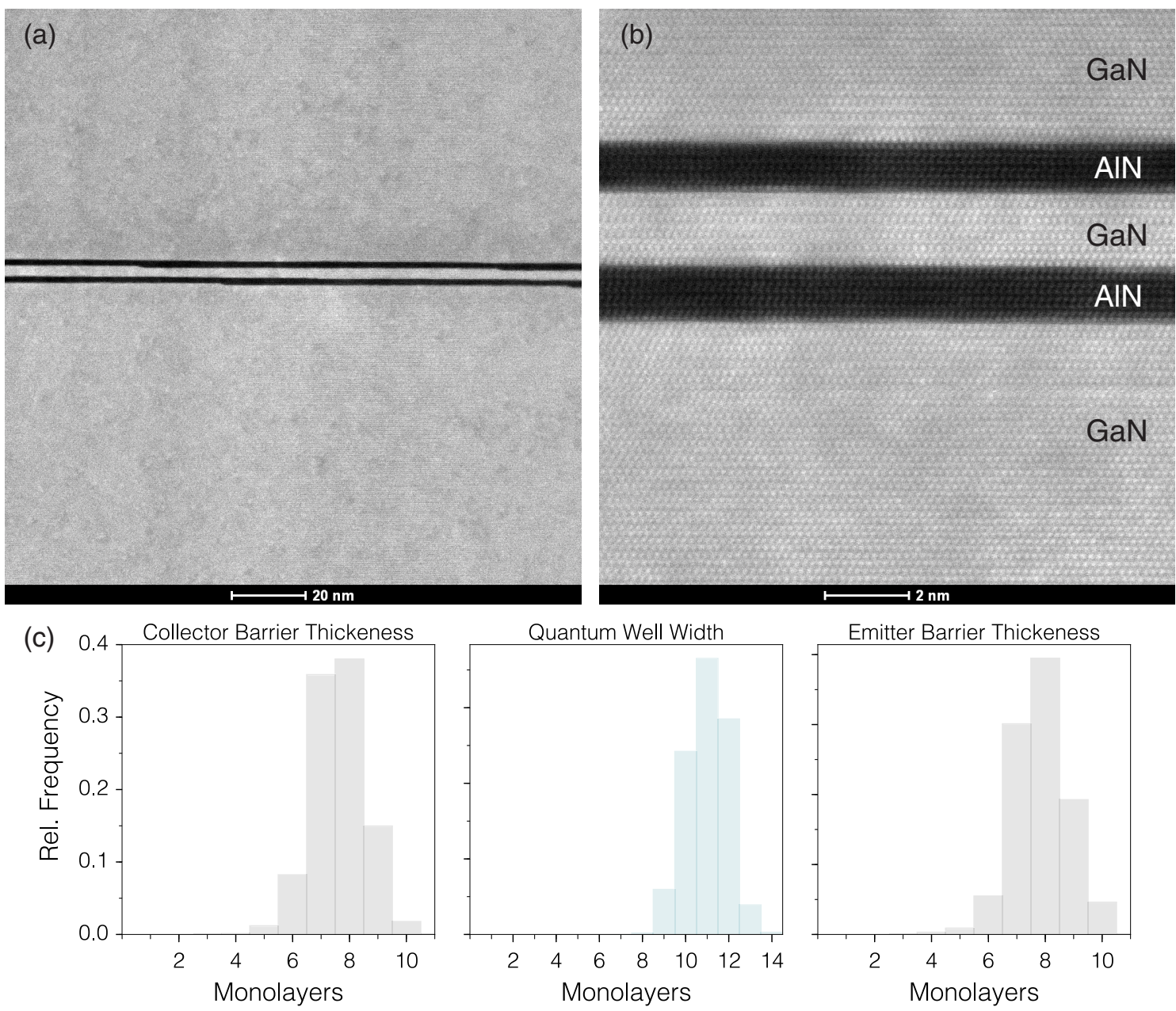

FIG. 4. STEM images of the GaN/AIN RTD heterostructure. (a) A scan over a large area shows the presence of fluctuations in the thickness of the ultrathin layers. (b) A smaller area scan shows the sharp transitions between the AlN barriers and the GaN quantum well. (c) Statistical distribution of the thickness of the barriers and width of the quantum well obtained by digital image processing of (a).

In order to further characterize the grown heterostructure, scanning transmission electron microscopy (STEM) has been employed to measure the thickness of the ultrathin layers. Figure 4(a) shows a cross-sectional STEM image of the double barriers extending over a distance of $\sim 160 \mathrm{~nm}$ perpendicular to the growth direction. The barriers and quantum well exhibit thickness fluctuations on the order of a few monolayers. The higher magnification image presented in Fig. 4(b) shows the detail of the sharp GaN/AlN interfaces over a smaller area. Digital image processing techniques have been applied to Fig. 4(a) to quantify the fluctuations in the thickness of the thin nitride layers. The statistical distribution is shown in Fig. 4(c); it is found that both barriers have a mean thickness of $8 \mathrm{ML}(\sim 2 \mathrm{~nm})$ with $1 \mathrm{ML}(\sim 0.25 \mathrm{~nm})$ fluctuations. The width of the well, however, presents a mean value of $11 \mathrm{ML}(\sim 2.75 \mathrm{~nm})$, which is $1 \mathrm{ML}$ less than the expected quantum well width. These variations can be incorporated in the electrostatic model developed in the next section so that more precise theoretical predictions are obtained.

\section{POLAR RTD MODEL}

An analytical electrostatic model is developed to capture the influence of the polarization fields on the resonant tunneling $I-V$ characteristics. The distribution of charge, electric field, and conduction band energy are calculated along the tunneling direction using the depletion approximation. Figures 5(a) and 5(b) display the results when the diode is in forward and reverse bias, respectively. The spontaneous polarization fields present in wurtzite III-nitride semiconductors as well as the piezoelectric contribution from the strained AlN barriers are considered. Sheets of effective polarization charge with densities $\pm \sigma_{\pi}$ are included at each interface, as shown in the charge diagrams of Figs. 5(a) and 5(b). The magnitude of the polarization field generated by these interface charges is given by $F_{\pi}=e \sigma_{\pi} / \epsilon_{s}$ ( $e$ is the electron charge and $\epsilon_{s}$ is the $\mathrm{GaN}$ dielectric constant).

Under equilibrium conditions [blue curve in Figs. 5(a) and 5(b)], a depletion region builds up in the collector side 


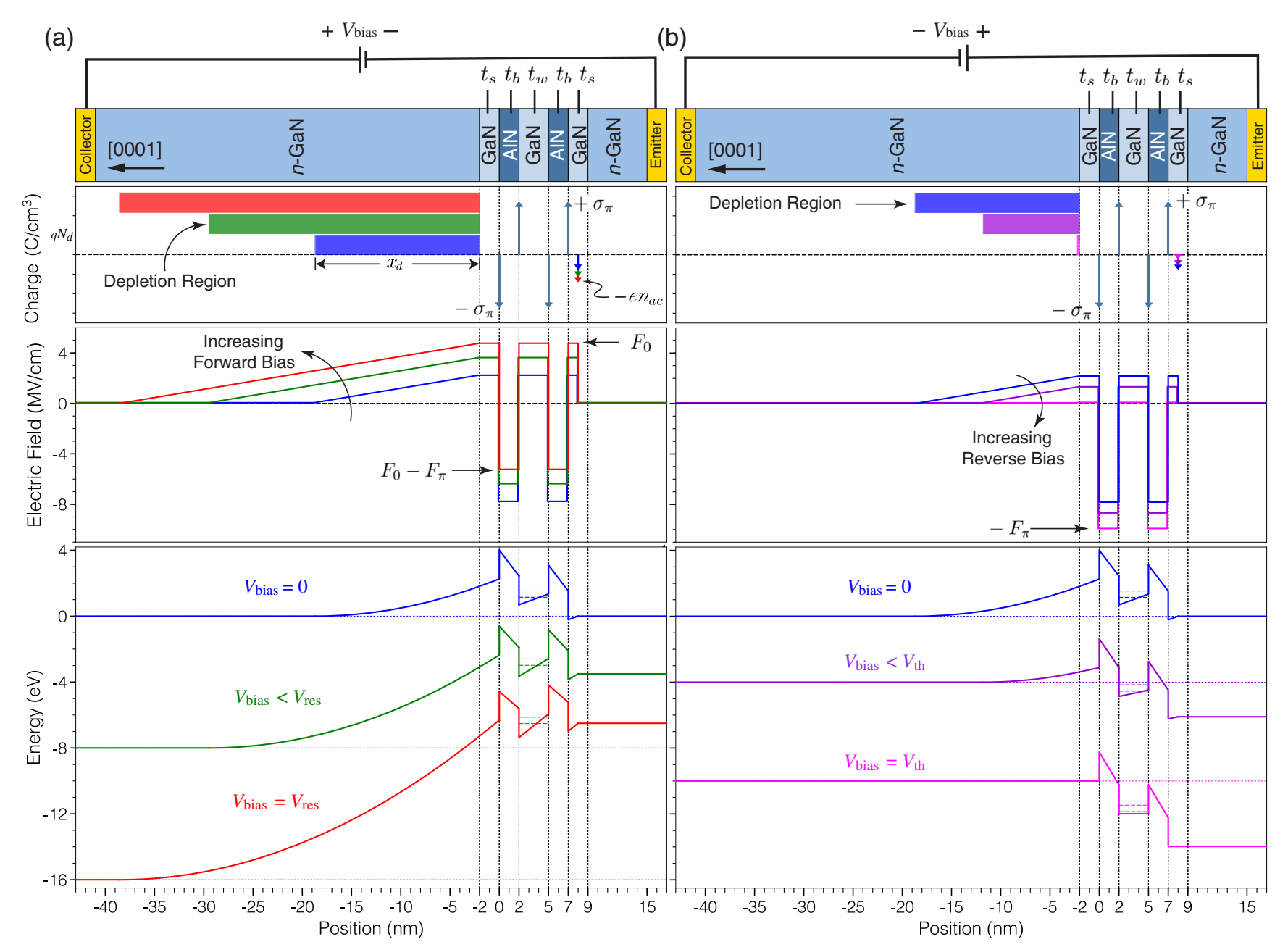

FIG. 5. Distribution of space charge, polarization charge, electric field, and electron energy in a GaN/AlN double-barrier heterostructure under forward and reverse bias. The polarity of the applied bias is that of the collector contact with the emitter as reference. (a) Under forward bias, the depletion region in the collector side becomes wider, causing an increment in the magnitude of the electric field $F_{0}$ inside the quantum well. As a consequence, the bound states shift towards lower energies due to the quantum confined Stark effect. Resonant transport arises when the energy of the ground state aligns with the bottom of the conduction band in the emitter side (red curve). (b) Under reverse bias, the collector depletion region becomes narrower while the electric field inside the quantum well tends to zero; at the same time, the electric fields within the barriers increase in magnitude. With a narrower depletion layer, electrons supporting the reverse current require less energy to reach the collector barrier and tunnel into the emitter region. A critical condition is achieved when the depletion region vanishes. Under this configuration, the threshold voltage $V_{\text {th }}$ is dropped across both barriers, which support an internal electric field equal to the polarization field $F_{\pi}$ (magenta curve).

while an accumulation well is induced next to the emitter barrier. The depletion width $x_{d}$ and the accumulation charge, modeled by the electron sheet density $-e n_{\mathrm{ac}}$, are both bias dependent. Thus, the electric field $F_{0}$ generated by the collector space charge is also bias dependent and will be constant within the collector spacer region. The polarization fields $F_{\pi}$ generated by the interface charges flip the sign of the electric field inside the barriers $-F_{0}-F_{\pi}$ is negative-while the electric field inside the GaN quantum well remains $F_{0}$ [see the electric field plots in Figs. 5(a) and 5(b)]. When a bias is applied, the space charge regions are modulated and the magnitude of the electric field $F_{0}$ is in turn modified. The relation between the applied bias and the internal electric fields $F_{0}$ and $F_{\pi}$ is given by the following analytical expression:

$V_{\text {bias }}=\frac{\epsilon_{s}}{2 e N_{d}} F_{0}^{2}+\left(t_{s}+2 t_{b}+t_{w}+t_{c}\right) F_{0}-2 t_{b} F_{\pi}$,

where $N_{d}$ is the dopant concentration in the collector side. All the thicknesses of the layers are shown in Fig. 5 and $t_{c}$ is the distance between the centroid of the accumulation layer and the emitter barrier. Using a Schrödinger-Poisson solver [36], we find $t_{c} \approx 1 \mathrm{~nm}$ under equilibrium conditions and we consider this value to be approximately constant. Thus, $F_{0}$ in Eq. (1) can be solved for any applied bias, since it is the sole unknown. 
Under forward bias, $F_{0}$ increases due to the larger amount of charge present in the accumulation and depletion regions. As a consequence, the electric field inside the well also increases and the quantum confined Stark effect shifts the energies of the bound states. Resonant conditions arise when the quantum well eigenenergies align with the bottom of the conduction band on the emitter side [red curve in Fig. 5(a)]. The energies of the bound states can be calculated within the framework of a finite quantum well perturbed by the electric field $F_{0}$ present in the well. Thus, if we label the unperturbed ground-state energy with $E_{0}$, solving for the electric field that satisfies the resonant condition gives

$$
F_{0}^{\mathrm{res}}=\frac{E_{0}+F_{\pi} e t_{b}}{e\left(\frac{t_{w}}{2}+t_{b}+t_{c}\right)} .
$$

Using this resonant electric field in Eq. (1) gives the theoretical resonant voltage $V_{\text {res }}$, under forward bias.

Moreover, insight into the reverse bias operation of III-nitride polar RTDs is also obtained from our model. As shown in Fig. 5(b), when a reverse bias is applied to the device, the electric field $F_{0}$ exhibits a decreasing trend. This is a result of the narrowing of the depletion layer in the collector region. As the reverse bias is increased, the electric field within the $\mathrm{GaN}$ layers tends to zero and the magnitude of the electric field inside the AlN barriers increases. As the depletion width gets narrower, the effective barrier seen by the conduction electrons on the collector side is reduced and the reverse bias current increases. A critical regime is achieved when $F_{0}=0$ and the depletion region is completely suppressed. Under these flat-band conditions, the overall voltage is dropped across both barriers. As a result, the electric field sustained by each of the barriers is exactly the polarization field $F_{\pi}$ [magenta curve in Fig. 5(b)]. Thus, for a RTD with symmetric barriers, we have this critical threshold voltage $V_{\text {th }}$ given by the simple expression:

$$
V_{\mathrm{th}}=-2 t_{b} F_{\pi} .
$$

As illustrated in Fig. 5(b), when the device is biased at this critical threshold voltage, electrons tunneling through the collector AlN barrier will support the overall reverse current. Since the collector barrier is under a high electric field $\left(F_{\pi} \sim 10 \mathrm{MV} / \mathrm{cm}\right)$, Fowler-Nordheim tunneling is expected to be the main conduction mechanism for voltages above this critical bias condition. This single-barrier tunneling transport regime contrasts with the double-barrier resonant tunneling mechanism supporting the current in forward bias. Thus, asymmetric current-voltage characteristics are expected in polar RTDs due to the presence of the built-in polarization fields.

Early studies on GaN/AlN RTDs found a strongly asymmetric $I-V$ in which the resonant peak was observed only in one bias direction $[9,13,43]$. Transfer matrix calculations by Sakr et al. showed the connection between the strong polarization fields and the asymmetric $I-V$ characteristics [44]. More recently, after repeatable III-nitride RTD behavior was achieved, the focus was mainly on the forward bias resonance [25-27,29]. It should be noted, however, that the internal polarization fields markedly manifest also in the reverse bias regime giving rise to a characteristic threshold voltage $V_{\text {th }}$ given by Eq. (3). This feature uniquely identifies a polar doublebarrier tunneling heterostructure and can be used to directly measure the internal polarization fields of polar materials as follows: if we consider a set of polar RTDs with different barrier thicknesses, the polarization discontinuity will be given by half the slope of the threshold voltage versus barrier thickness, assuming symmetric doublebarrier heterostructures.

To conclusively demonstrate the validity of Eq. (3), a set of double-barrier GaN/AlN RTDs with variable barrier thickness is grown and fabricated. The device structures feature a constant doping concentration of $N_{d} \sim 2 \times 10^{19} \mathrm{~cm}^{-3}$ and spacers of nominal thicknesses 10 and $6 \mathrm{~nm}$ in the emitter and collector regions, respectively. Repeatable roomtemperature NDC is measured in each of these samples featuring AlN barrier thickness between 1.5 and $2.4 \mathrm{~nm}$. It should be pointed out that none of these RTDs, grown at a higher substrate temperature, exhibit any voltage or current shift in the resonant peak. This robust resonant tunneling transport, measured at room temperature across different RTD designs, allows us to accurately measure the magnitude of the polarization fields present in the GaN/AlN doublebarrier heterostructure.

The critical threshold voltage $V_{\text {th }}$ measured in this set of samples is shown in Fig. 6 as a function of barrier thickness. X-ray diffraction is employed to measure the thickness of the barriers by fitting the intensity of the diffracted $\mathrm{x}$ rays with a theoretical model. As expected from Eq. (3), the critical threshold voltages fall in a straight line with a characteristic slope $-2 F_{\pi}$. By linearly fitting the experimental values in Fig. 6, we obtain the magnitude of the overall polarization field to be $F_{\pi}=11.0 \pm 0.3 \mathrm{MV} / \mathrm{cm}$ for AlN pseudomorphically strained on GaN. This value agrees very well with the theoretical predictions by Ambacher et al. [45] and the experimental value of $10.9 \mathrm{MV} / \mathrm{cm}$ extracted from an AlN/GaN high electron mobility transistor [46].

Since the polarization fields play a crucial role in the design of electronic and photonic devices, they have been recently reexamined to obtain a more accurate theoretical description [47]. However, there exists a considerable spread in the reported values of the polarization field discontinuities both in $\mathrm{GaN} / \mathrm{InGaN}$ and $\mathrm{GaN} / \mathrm{AlGaN}$ heterojunctions. In this context, we introduce here a completely new method to directly measure the polarization fields present in any polar heterojunction. 


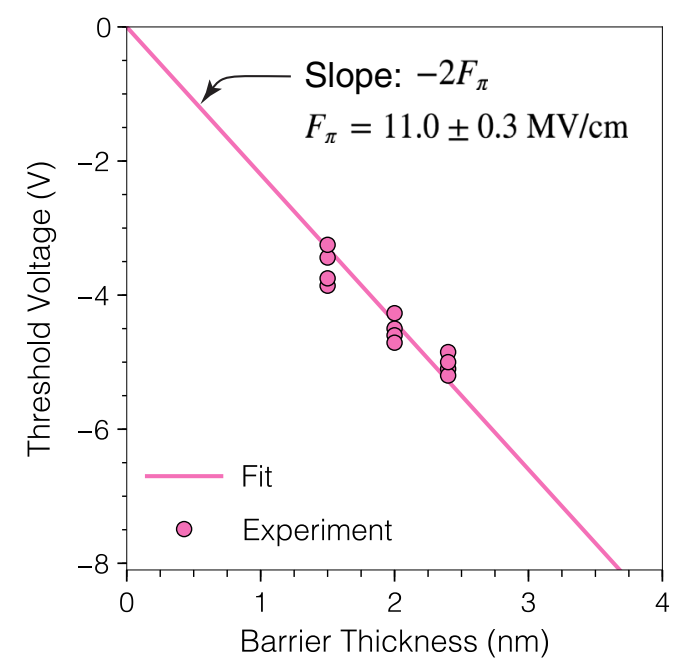

FIG. 6. Critical threshold voltage $V_{\text {th }}$ as a function of barrier thickness for a set of RTDs exhibiting NDC at room temperature. Several devices were measured on each of the fabricated samples. All the device structures feature a constant doping concentration $N_{d} \sim 2 \times 10^{19} \mathrm{~cm}^{-3}$ and spacers of thicknesses 10 and $6 \mathrm{~nm}$ in the emitter and collector region, respectively. By linearly fitting the experimental data using Eq. (3), a polarization field $F_{\pi}=$ $11.0 \pm 0.3 \mathrm{MV} / \mathrm{cm}$ is extracted. This result agrees very well with theoretical and experimental values previously published $[45,46]$.

\section{POLAR RTD AT RESONANCE}

The influence of the internal piezoelectric polarization fields on the resonant peak of a double-barrier heterostructure was recognized and studied initially in InGaAs/InAlAs [48] and InGaAs/AlGaAs [49] RTDs. The strain-induced polarization fields on these heterostructures are, however, much weaker compared to the spontaneous and piezoelectric polarization fields present in III-nitride heterostructures. To study the effects of these stronger polarization fields, we can consider a general polar RTD biased at resonance. Figure 7(a) shows in particular the band diagram of a GaN/AlN double-barrier RTD. When the device is baised at resonance, the electron transmission will be enhanced, exhibiting a maximum value $-T_{\max }$-at the resonant state energy $E_{r}$. The transmission probability around the resonant energy can be approximated by the Breit-Wigner function [50]:

$$
T(E)=T_{\max } \frac{(\Delta E)^{2}}{\left(E-E_{r}\right)^{2}+(\Delta E)^{2}},
$$

where $\Delta E$ is half the full width at half maximum (FWHM) of the resonant transmission peak. Thus, the tunneling current at the resonant configuration can be calculated by using this last expression in the Tsu-Esaki integral. In the case of a narrow resonance, a simple expression can be obtained for the resonant tunneling current density [51]:

$$
J_{\text {res }} \simeq \frac{e m^{*}}{2 \pi \hbar^{3}} T_{\max }(\Delta E) E_{F},
$$

with $E_{F}$ being the Fermi energy in the emitter side and $m^{*}$ the effective mass. $T_{\max }$ can be expressed in terms of the single-barrier transmission coefficients of the emitter and collector barriers, $T_{E}$ and $T_{C}$, respectively:

$$
T_{\max }=\frac{4 T_{E} T_{C}}{\left(T_{E}+T_{C}\right)^{2}}
$$

A semiclassical picture can be employed to calculate the FWHM of the resonant transmission function; thus, $\Delta E$ is given by [52]

$$
\Delta E \approx \frac{\hbar}{2} \frac{\sqrt{2 E_{r}^{\prime} / m_{w}^{*}}}{2 t_{w}}\left(T_{E}+T_{C}\right)
$$

where $E_{r}^{\prime}$ is the resonant energy referred to the bottom of the well and $m_{w}^{*}$ is the electron effective mass within the well. Expressions for single-barrier tunneling coefficients $T_{i}$ can be obtained by employing the Wentzel-KramersBrillouin formalism:

$$
T_{i} \sim \exp \left(-\frac{4 \sqrt{2 m_{b}^{*}}}{3 \hbar} \frac{\left(\Phi_{i}^{(2)}\right)^{3 / 2}-\left(\Phi_{i}^{(1)}\right)^{3 / 2}}{e\left(F_{\pi}-F_{0}^{\text {res }}\right)}\right) .
$$

The barrier heights $\Phi_{i}^{(1)}$ and $\Phi_{i}^{(2)}$ are labeled in the band diagram of Fig. 7(a), which shows the conduction band profile at resonance. Thus, Eqs. (5), (6), (7), and (8) can be employed to compute the resonant tunneling current for any particular double-barrier RTD design.

The calculated resonant tunneling current $J_{\text {res }}$ is shown in Fig. 7(b) as a function of the tunneling barrier thickness. The experimental resonant currents reported in the same figure are measured in the set of RTDs introduced in the previous section. These devices feature variable barrier thickness of 1.5, 2.0, and $2.4 \mathrm{~nm}$, obtained by fitting their $\mathrm{x}$-ray diffraction patterns with a theoretical model. The polarization discontinuity extracted in the previous section is employed in Eq. (8) to calculate the expected resonant tunneling current. According to our model, the RTD resonant current is expected to increase by approximately 2 orders of magnitude when the barriers are thinned down by $1 \mathrm{~nm}$. This trend is experimentally verified since RTDs featuring AlN barriers of 1.5 and $2.4 \mathrm{~nm}$ drive resonant currents of $\sim 2.5 \times 10^{2}$ and $\sim 2.6 \times 10^{4} \mathrm{~A} / \mathrm{cm}^{2}$, respectively.

As is shown in the TEM analysis of the previous section, single ML fluctuations might also be present in the tunneling barriers of these samples. These fluctuations are indicated by the error bar in Fig. 7(b). A detailed discussion of the $I-V$ curves and magnitude of their NDC will be presented elsewhere. However, these results further confirm the control exerted by the AlN barriers on the magnitude of the resonant tunneling current. 

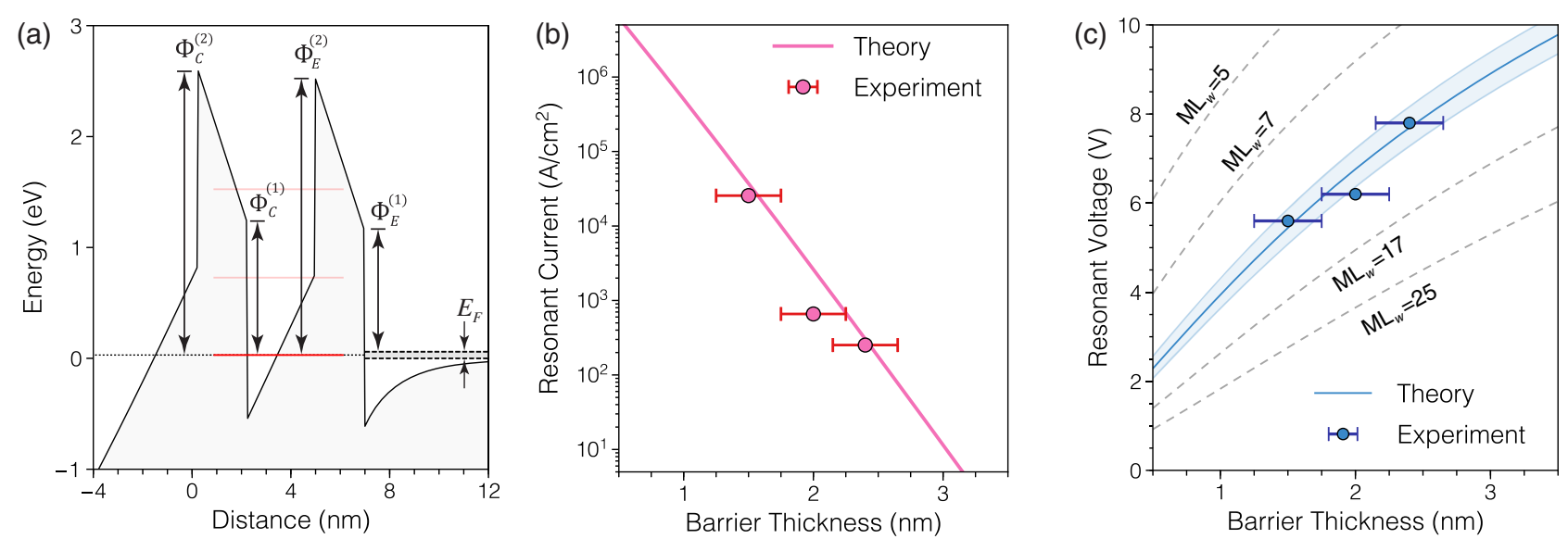

FIG. 7. (a) Band diagram of a GaN/AIN double-barrier heterostructure at resonance showing the various energy barriers considered in the calculation of the resonant tunneling current. (b) Resonant tunneling current as a function of barrier thickness predicted by our analytical model. The experimental values were measured in a set of RTDs with variable barrier thickness exhibiting NDC at room temperature. The heterostructures feature a doping concentration $N_{d} \sim 2 \times 10^{19} \mathrm{~cm}^{-3}$ and spacers of thickness 10 and $6 \mathrm{~nm}$ in the emitter and collector region, respectively. The error bars indicate $\pm 1-\mathrm{ML}( \pm 0.25-\mathrm{nm})$ fluctuations in the AlN barriers. (c) Resonant voltage $V_{\text {res }}$ as a function of the barrier thickness and width of the quantum well in monolayers $\left(\mathrm{ML}_{w}\right)$ for a GaN/AlN RTD. The shaded region shows the effect of 1-ML fluctuations around an 11-ML GaN well. The experimental values were measured at room temperature in the same set of devices reported in (b).

In addition to the peak tunneling current, an analytical expression can also be derived for the peak resonant voltage of the $I-V$ curve. As we mention in the previous section, Eqs. (1) and (2) can be used to compute the resonant voltage $V_{\text {res }}$, which is a function of both barrier thickness and quantum well width. Figure 7(c) displays the values of $V_{\text {res }}$ predicted by our model using the magnitude of the polarization field measured in our devices: $F_{\pi}=11.0 \mathrm{MV} / \mathrm{cm}$. The experimental values included in this figure were measured at room temperature in the same set of devices reported in Fig. 7(b). The horizontal error bars indicate the probable fluctuations in barrier thickness for each fabricated sample. In addition, the shaded region in Fig. 7(c) indicates the expected variation in the resonant voltage due to 1-ML fluctuations in the width of the quantum well. Thus, experiments and calculations show a fairly good agreement, suggesting that our model can be used to design a polar RTD with a desired resonant voltage and peak resonant tunneling current.

Looking forward, devices with larger peak-to-valley current ratios will be required for various practical applications. In this sense, enhancing adatom mobilities and using low growth rates can contribute towards reducing defect formation and obtaining atomically smooth interfaces, thus minimizing scattering processes that increase the magnitude of the valley current. On the other hand, the various effects of the built-in polarization fields can be suppressed by growing heterostructures on the nonpolar and semipolar planes of III-nitride crystals. A few reports have assessed the performance of $\mathrm{GaN} / \mathrm{Al}(\mathrm{Ga}) \mathrm{N}$ RTDs grown along the [11 100$]$ direction; however, this transport regime still remains largely unexplored $[53,54]$.

\section{CONCLUSIONS}

In conclusion, we experimentally and theoretically investigate the current-voltage characteristics of GaN/AlN resonant tunneling diodes. Various double-barrier heterostructure designs are grown by MBE and fabricated into diodes. The repeatable resonant tunneling transport in our devices has enabled us to identify and explain the origin of various new features that characterize quantum transport across a general polar double-barrier RTD.

It is shown that reverse bias operation of polar RTDs is governed by single-barrier tunneling transport and characterized by a well-defined threshold voltage that is intimately linked to the internal polarization fields. From this result, we realize that the threshold voltage can be used as a sensitive measure of the internal polarization fields present in any polar double-barrier tunneling heterostructure.

A model for polar resonant tunneling diodes is also developed and analytical expressions for the forward resonant voltage and resonant tunneling current are derived. These parameters are both intimately linked to the magnitude of the internal polarization fields, and its experimental values, measured in III-nitride RTDs, agree fairly well with the theoretical predictions of our model.

Subsequent measurements show the robustness of the negative differential conductance at room temperature. Moreover, resonant tunneling transport via the ground state and first excited state over a wide temperature window is demonstrated for the first time in III-nitride RTDs. These findings represent a significant step forward in resonant tunneling, intersubband-based physics, and III-nitride quantum devices. 


\section{ACKNOWLEDGMENTS}

The authors would like to acknowledge the support from the Office of Naval Research under the DATE MURI Program (Contract No. N00014-11-10721, program manager Dr. Paul Maki) and from the National Science Foundation under the NSF-DMREF Program (Contract No. 1534303, program manager Dr. John Schlueter).

[1] R. Tsu and L. Esaki, Tunneling in a Finite Superlattice, Appl. Phys. Lett. 22, 562 (1973).

[2] R. Kohler, A. Tredicucci, F. Beltram, H. E. Beere, E. H. Linfield, A. G. Davies, D. A. Ritchie, R. C. Iotti, and F. Rossi, Terahertz Semiconductor-Heterostructure Laser, Nature (London) 417, 156 (2002).

[3] M. A. Belkin and F. Capasso, New Frontiers in Quantum Cascade Lasers: High Performance Room Temperature Terahertz Sources, Phys. Scr. 90, 118002 (2015).

[4] E. R. Brown, T. C. L. G. Sollner, C. D. Parker, W. D. Goodhue, and C. L. Chen, Oscillations up to $420 \mathrm{GHz}$ in GaAs/AlAs Resonant Tunneling Diodes, Appl. Phys. Lett. 55, 1777 (1989).

[5] M. Hangyo, Development and Future Prospects of Terahertz Technology, Jpn. J. Appl. Phys. 54, 120101 (2015).

[6] K. Kishino, A. Kikuchi, H. Kanazawa, and T. Tachibana, Intersubband Transition in $(\mathrm{GaN})_{m} /(\mathrm{AlN})_{n}$ Superlattices in the Wavelength Range from 1.08 to $1.61 \mu \mathrm{m}$, Appl. Phys. Lett. 81, 1234 (2002).

[7] M. Tchernycheva, L. Nevou, L. Doyennette, F. H. Julien, E. Warde, F. Guillot, E. Monroy, E. Bellet-Amalric, T. Remmele, and M. Albrecht, Systematic Experimental and Theoretical Investigation of Intersubband Absorption in GaN/AlN Quantum Wells, Phys. Rev. B 73, 125347 (2006).

[8] M. A. Belkin, Q. J. Wang, C. Pflugl, A. Belyanin, S. P. Khanna, A. G. Davies, E. H. Linfield, and F. Capasso, HighTemperature Operation of Terahertz Quantum Cascade Laser Sources, IEEE J. Sel. Top. Quantum Electron. 15, 952 (2009).

[9] C. T. Foxon, S. V. Novikov, A. E. Belyaev, L. X. Zhao, O. Makarovsky, D. J. Walker, L. Eaves, R. I. Dykeman, S. V. Danylyuk, S. A. Vitusevich, M. J. Kappers, J. S. Barnard, and C.J. Humphreys, Current-Voltage Instabilities in GaN/AlGaN Resonant Tunnelling Structures, Phys. Status Solidi C 0, 2389 (2003).

[10] M. Boucherit, A. Soltani, E. Monroy, M. Rousseau, D. Deresmes, M. Berthe, C. Durand, and J.-C. De Jaeger, Investigation of the Negative Differential Resistance Reproducibility in AlN/GaN Double-Barrier Resonant Tunnelling Diodes, Appl. Phys. Lett. 99, 182109 (2011).

[11] M. Nagase and T. Tokizaki, Bistability Characteristics of GaN/AlN Resonant Tunneling Diodes Caused by Intersubband Transition and Electron Accumulation in Quantum Well, IEEE Trans. Electron Devices 61, 1321 (2014).

[12] M. Nagase, T. Takahashi, and M. Shimizu, Investigating the Bistability Characteristics of GaN/AlN Resonant Tunneling Diodes for Ultrafast Nonvolatile Memory, Jpn. J. Appl. Phys. 54, 034201 (2015).
[13] A. Kikuchi, R. Bannai, K. Kishino, C.-M. Lee, and J.-I. Chyi, AlN/GaN Double-Barrier Resonant Tunneling Diodes Grown by rf-Plasma-Assisted Molecular-Beam Epitaxy, Appl. Phys. Lett. 81, 1729 (2002).

[14] C. Bayram, Z. Vashaei, and M. Razeghi, AlN/GaN Double-Barrier Resonant Tunneling Diodes Grown by Metal-Organic Chemical Vapor Deposition, Appl. Phys. Lett. 96, 042103 (2010).

[15] C. Bayram, Z. Vashaei, and M. Razeghi, Reliability in Room-Temperature Negative Differential Resistance Characteristics of Low-Aluminum Content AlGaN/GaN Double-Barrier Resonant Tunneling Diodes, Appl. Phys. Lett. 97, 181109 (2010).

[16] R. Songmuang, G. Katsaros, E. Monroy, P. Spathis, C. Bougerol, M. Mongillo, and S. De Franceschi, Quantum Transport in GaN/AIN Double-Barrier Heterostructure Nanowires, Nano Lett. 10, 3545 (2010).

[17] Y. Shao, S. D. Carnevale, A. T. M. G. Sarwar, R. C. Myers, and W. Lu, Single Nanowire AlN/GaN Double Barrier Resonant Tunneling Diodes with Bipolar Tunneling at Room and Cryogenic Temperatures, J. Vac. Sci. Technol., B 31, 06FA03 (2013).

[18] A. Kikuchi, R. Bannai, and K. Kishino, AlGaN Resonant Tunneling Diodes Grown by rf-MBE, Phys. Status Solidi A 188, 187 (2001).

[19] A. E. Belyaev, O. Makarovsky, D. J. Walker, L. Eaves, C. T. Foxon, S. V. Novikov, L. X. Zhao, R. I. Dykeman, S. V. Danylyuk, S. A. Vitusevich, M. J. Kappers, J. S. Barnard, and C. J. Humphreys, Resonance and Current Instabilities in AlN/GaN Resonant Tunnelling Diodes, Physica (Amsterdam) 21E, 752 (2004).

[20] S. Golka, C. Pflügl, W. Schrenk, G. Strasser, C. Skierbiszewski, M. Siekacz, I. Grzegory, and S. Porowski, Negative Differential Resistance in Dislocation-Free GaN/AlGaN Double-Barrier Diodes Grown on Bulk GaN, Appl. Phys. Lett. 88, 172106 (2006).

[21] A. M. Kurakin, S. A. Vitusevich, S. V. Danylyuk, A. V. Naumov, C. T. Foxon, S. V. Novikov, N. Klein, H. Lüth, and A.E. Belyaev, Capacitance Characterization of AlN/GaN Double-Barrier Resonant Tunneling Diodes, Phys. Status Solidi C 3, 2265 (2006).

[22] M. V. Petrychuk, A. E. Belyaev, A. M. Kurakin, S. V. Danylyuk, N. Klein, and S. A. Vitusevich, Mechanisms of Current Formation in Resonant Tunneling AlN/GaN Heterostructures, Appl. Phys. Lett. 91, 222112 (2007).

[23] C. Bayram, Z. Vashaei, and M. Razeghi, Room Temperature Negative Differential Resistance Characteristics of Polar III-Nitride Resonant Tunneling Diodes, Appl. Phys. Lett. 97, 092104 (2010).

[24] S. Sakr, Y. Kotsar, M. Tchernycheva, E. Warde, N. Isac, E. Monroy, and F. H. Julien, Resonant Tunneling Transport in $a$ GaN/AlN Multiple-Quantum-Well Structure, Appl. Phys. Express 5, 052203 (2012).

[25] D. Li, L. Tang, C. Edmunds, J. Shao, G. Gardner, M. J. Manfra, and O. Malis, Repeatable Low-Temperature Negative-Differential Resistance from $\mathrm{Al}_{0.18} \mathrm{Ga}_{0.82} \mathrm{~N} / \mathrm{GaN}$ Resonant Tunneling Diodes Grown by Molecular-Beam Epitaxy on Free-Standing GaN Substrates, Appl. Phys. Lett. 100, 252105 (2012). 
[26] D. Li, J. Shao, L. Tang, C. Edmunds, G. Gardner, M. J. Manfra, and O. Malis, Temperature-Dependence of Negative Differential Resistance in GaN/AlGaN Resonant Tunneling Structures, Semicond. Sci. Technol. 28, 074024 (2013).

[27] A. Grier, A. Valavanis, C. Edmunds, J. Shao, J. D. Cooper, G. Gardner, M. J. Manfra, O. Malis, D. Indjin, Z. Ikonić, and P. Harrison, Coherent Vertical Electron Transport and Interface Roughness Effects in $\mathrm{AlGaN} / \mathrm{GaN}$ Intersubband Devices, J. Appl. Phys. 118, 224308 (2015).

[28] J. Encomendero, F. Afroz Faria, S. M. Islam, V. Protasenko, S. Rouvimov, P. Fay, D. Jena, and H. G. Xing, Repeatable Room Temperature Negative Differential Conductance in GaN/AlN Resonant Tunneling Diodes, arXiv:1606.08100.

[29] T. A. Growden, D. F. Storm, W. Zhang, E. R. Brown, D. J. Meyer, P. Fakhimi, and P. R. Berger, Highly Repeatable Room Temperature Negative Differential Resistance in AlN/GaN Resonant Tunneling Diodes Grown by Molecular Beam Epitaxy, Appl. Phys. Lett. 109, 083504 (2016).

[30] C. Adelmann, J. Brault, G. Mula, B. Daudin, L. Lymperakis, and J. Neugebauer, Gallium Adsorption on (0001) GaN Surfaces, Phys. Rev. B 67, 165419 (2003).

[31] C. Adelmann, J. Brault, D. Jalabert, P. Gentile, H. Mariette, G. Mula, and B. Daudin, Dynamically Stable Gallium Surface Coverages during Plasma-Assisted MolecularBeam Epitaxy of (0001) GaN, J. Appl. Phys. 91, 9638 (2002).

[32] P. K. Kandaswamy, F. Guillot, E. Bellet-Amalric, E. Monroy, L. Nevou, M. Tchernycheva, A. Michon, F. H. Julien, E. Baumann, F. R. Giorgetta, D. Hofstetter, T. Remmele, M. Albrecht, S. Birner, and Le Si Dang, GaN/AlN ShortPeriod Superlattices for Intersubband Optoelectronics: A Systematic Study of Their Epitaxial Growth, Design, and Performance, J. Appl. Phys. 104, 093501 (2008).

[33] E. Iliopoulos and T. D. Moustakas, Growth Kinetics of AlGaN Films by Plasma-Assisted Molecular-Beam Epitaxy, Appl. Phys. Lett. 81, 295 (2002).

[34] S. Leconte, L. Gerrer, and E. Monroy, Electronic Transport through GaN/AlN Single Barriers: Effect of Polarisation and Dislocations, Microelectron. J. 40, 339 (2009).

[35] Y. Cao and D. Jena, High-Mobility Window for TwoDimensional Electron Gases at Ultrathin AlN/GaN Heterojunctions, Appl. Phys. Lett. 90, 182112 (2007).

[36] I. H. Tan, G. L. Snider, L. D. Chang, and E. L. Hu, A Self Consistent Solution of Schrödinger-Poisson Equations Using a Nonuniform Mesh, J. Appl. Phys. 68, 4071 (1990).

[37] J. S. Wu, C. Y. Chang, C. P. Lee, K. H. Chang, D. G. Liu, and D. C. Liou, Resonant Tunneling of Electrons from Quantized Levels in the Accumulation Layer of Double Barrier Heterostructures, Appl. Phys. Lett. 57, 2311 (1990).

[38] M. Buchanan, H. C. Liu, T. G. Powell, and Z. R. Wasilewski, Magneto-Resonant Tunneling from a Lightly Doped Contact Region Interacting with Quasi-Two-Dimensional States in an Accumulation Layer, J. Appl. Phys. 68, 4313 (1990).

[39] V. J. Goldman, D. C. Tsui, J. E. Cunningham, and W. T. Tsang, Transport in Double-Barrier Resonant Tunneling Structures, J. Appl. Phys. 61, 2693 (1987).

[40] J. S. Wu, C.-Y. Chang, C.-P. Lee, Y.-H. Wang, and F. Kai, Origin of the Enhancement of Negative Differential Resistance at Low Temperatures in Double-Barrier Resonant Tunneling Structures, IEEE Electron Device Lett. 10, 301 (1989).
[41] G. D. Shen, D. X. Xu, M. Willander, and G. V. Hansson, in Proceedings of the IEEE/Cornell Conference on Advanced Concepts in High Speed Semiconductor Devices and Circuits, 1991 (IEEE, New York, 1991) pp. 84-93, http:// ieeexplore.ieee.org/document/170037/.

[42] R. Yan, S. Fathipour, Y. Han, B. Song, S. Xiao, M. Li, N. Ma, V. Protasenko, D. A. Muller, D. Jena, and H. G. Xing, Esaki Diodes in van der Waals Heterojunctions with Broken-Gap Energy Band Alignment, Nano Lett. 15, 5791 (2015).

[43] M. Hermann, E. Monroy, A. Helman, B. Baur, M. Albrecht, B. Daudin, O. Ambacher, M. Stutzmann, and M. Eickhoff, Vertical Transport in Group III-Nitride Heterostructures and Application in AlN/GaN Resonant Tunneling Diodes, Phys. Status Solidi C 1, 2210 (2004).

[44] S. Sakr, E. Warde, M. Tchernycheva, and F. H. Julien, Ballistic Transport in GaN/AlGaN Resonant Tunneling Diodes, J. Appl. Phys. 109, 023717 (2011).

[45] O. Ambacher, B. Foutz, J. Smart, J. R. Shealy, N. G. Weimann, K. Chu, M. Murphy, A. J. Sierakowski, W. J. Schaff, L. F. Eastman, R. Dimitrov, A. Mitchell, and M. Stutzmann, Two Dimensional Electron Gases Induced by Spontaneous and Piezoelectric Polarization in Undoped and Doped $\mathrm{AlGaN} / \mathrm{GaN}$ Heterostructures, J. Appl. Phys. 87, 334 (2000).

[46] K. Jeganathan, T. Ide, M. Shimizu, and H. Okumura, TwoDimensional Electron Gases Induced by Polarization Charges in AlN/GaN Heterostructure Grown by PlasmaAssisted Molecular-Beam Epitaxy, J. Appl. Phys. 94, 3260 (2003).

[47] C. E. Dreyer, A. Janotti, C. G. Van de Walle, and D. Vanderbilt, Correct Implementation of Polarization Constants in Wurtzite Materials and Impact on III-Nitrides, Phys. Rev. X 6, 021038 (2016).

[48] J. M. Hernández, I. Izpura, E. Calleja, and E. Muoz, Piezoelectric Induced Current Asymmetry in [111] InGaAs/InAlAs Resonant Tunneling Diodes for Microwave Mixing, Appl. Phys. Lett. 63, 773 (1993).

[49] I. H. Campbell, M. D. Joswick, D. L. Smith, and R. H. Miles, Observation of Piezoelectric Effects in Strained Resonant Tunneling Structures Grown on (111)B GaAs, Appl. Phys. Lett. 66, 988 (1995).

[50] H.C. Liu and G.C. Aers, Resonant Tunneling through One-, Two-, and Three-Dimensionally Confined Quantum Wells, J. Appl. Phys. 65, 4908 (1989).

[51] D. D. Coon and H. C. Liu, Frequency Limit of Double Barrier Resonant Tunneling Oscillators, Appl. Phys. Lett. 49, 94 (1986).

[52] R. A. Kiehl and T.C.L. Gerhard Sollner, High Speed Heterostructure Devices (Academic Press, New York, 1994).

[53] S. D. Carnevale, C. Marginean, P. J. Phillips, T. F. Kent, A. T. M. G. Sarwar, M. J. Mills, and R. C. Myers, Coaxial Nanowire Resonant Tunneling Diodes from Non-Polar AlN/GaN on Silicon, Appl. Phys. Lett. 100, 142115 (2012).

[54] C. Bayram, D. K. Sadana, Z. Vashaei, and M. Razeghi, Reliable GaN-Based Resonant Tunneling Diodes with Reproducible Room-Temperature Negative Differential Resistance, Proc. SPIE Int. Soc. Opt. Eng. 8268, 826827 (2012). 